\title{
Enantioselective Synthesis of [3]-Ladderanol through a Late-Stage Organocatalytic Desymmetrization
}

\author{
Sayan Ray, Subhajit Mondal and Santanu Mukherjee* \\ Department of Organic Chemistry, Indian Institute of Science \\ Bangalore 560012 (India) \\ E-mail: sm@iisc.ac.in
}

\begin{abstract}
Ladderane phospholipids, with their unusual ladder-like arrangement of fused cyclobutane rings, represent an architecturally unique class of natural products. However, despite their fascinating structure and other necessary impetus, only a few synthetic studies of these molecules have been reported so far. We have now devised a concise synthesis of [3]-ladderanol, a component of ladderane phospholipids, using an organocatalytic enantioselective desymmetrizing formal $\mathrm{C}\left(\mathrm{sp}^{2}\right)-\mathrm{H}$ alkylation. Our synthetic strategy rests on a late-stage introduction of chirality, thus allowing facile access to both the enantiomers of [3]-ladderanol as well as its analog. This is the first time a desymmetrization strategy is applied to the synthesis of [3]-ladderanol. The scope of this desymmetrizing $\mathrm{C}\left(\mathrm{sp}^{2}\right)-\mathrm{H}$ alkylation of meso-cyclobutane fused cyclohexenediones is also presented.
\end{abstract}

\section{Introduction}

Architecturally unique natural products have always captivated organic chemists and inspired the development of new synthetic tools for achieving those targets. ${ }^{[1]}$ Isolation of ladderane phospholipids 1-2 (Figure 1) in 2002 from anerobic ammonium oxidizing (anammox) bacteria revealed such never-seen-before structural features in nature. ${ }^{[2]}$ These molecules were named so due to the presence of highly unusual ladder-like arrangements of concatenated cyclobutane rings. ${ }^{[3]}$

The perceived function of the anammox bacteria is as fascinating as the structure of these lipids. In a large intracellular compartment of these bacteria, known as the anammoxosome, ammonium and nitrite are coupled to produce dinitrogen in an overall exergonic reaction called the anammox process. ${ }^{[4]}$ The presence of ladderane phospholipids into the membrane of anammoxosome imparts unusual density, which helps in maintaining a $\mathrm{pH}$ gradient across the membrane that drives ATP synthesis. ${ }^{[5]}$

Due to the slow growth rate of the producing organism and the inherent difficulty in purifying such complex lipid mixtures, ${ }^{[6]}$ laboratory synthesis remains the primary source of ladderane phospholipids for studying the relationship between their structure and biological function.

As shown in Figure 1, these ladderane phospholipids are composed of either (+)-[3]-ladderanol (3) or a mixture of (+)-[3]ladderanol (3) and (-)-[5]-ladderanoic acid (4)..$^{7]}$ While several strategies were conceived for the construction of non-natural ladderanes prior to $2002,{ }^{[8]}$ only a few research groups have invested into the synthesis of naturally occurring ladderane phospholipids so far. ${ }^{[9]}$ [5]-Ladderanoic acid (4) was the first member of the ladderane family of natural products to be synthesized. The pioneering synthesis by Corey et al. in 2004 resulted 4 in racemic form. ${ }^{[10]}$ Soon after, the same group disclosed the synthesis of $(+)$-[5]ladderanoic acid [(+)-4] using a diastereoselective photochemical [2+2]-cycloaddition. ${ }^{[11]}$ More than a decade after these initial reports, Burns $^{[12]}$ and Brown ${ }^{[13]}$ groups synthesized both [3]-ladderanol (3) and [5]-ladderanoic acid (4). Burns et al. went on to assemble (+)-3 and (-)-4 into phospholipid 1 and established its absolute configuration. ${ }^{[12]}$

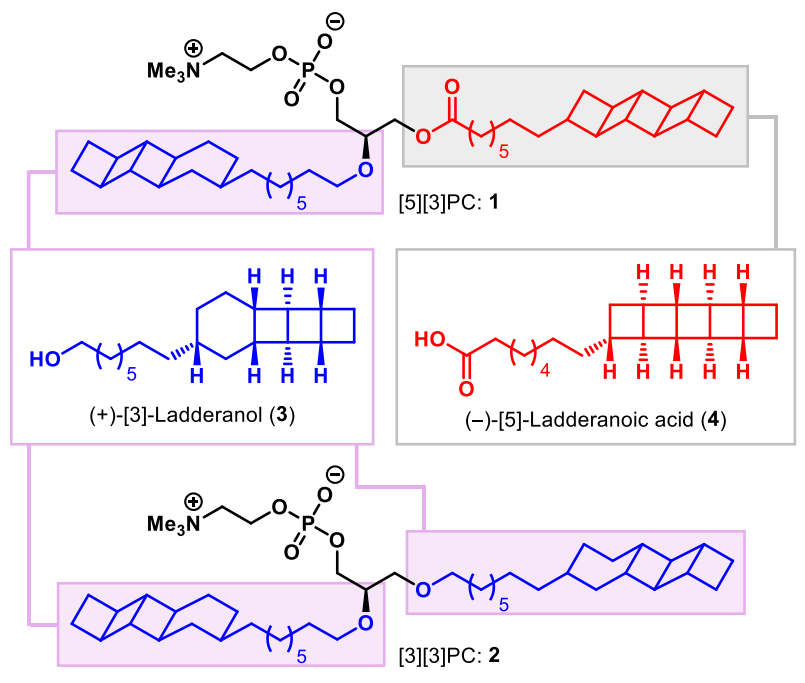

Figure 1. Natural ladderane phospholipids and their tail groups. PC denotes phosphatidylcholine. 
While both Burns' and Brown's elegant syntheses of [5]-ladderanoic acid (4) made use of the symmetry of the [5]-ladderane core itself ${ }^{[12]}$ or its lower member, ${ }^{[13 a]}$ a desymmetrization route to [3]-ladderanol (3) has never been realized. Burns' synthesis of $(+)-3$ begins with a lipase-resolved enantiopure dibromodio[[12] whereas Brown's strategy to (-)-3 hinges on an organocatalytic enantioselective isomerization of a deconjugated alkynyl ketone, followed by an intramolecular chirality transfer [2+2]-cycloaddition of the resulting chiral allenone ${ }^{[13 \mathrm{~b}]}$ (Scheme $1 \mathrm{~A}$ ). In both these syntheses, the enantioinduction takes place at the early phase of the synthetic route and is carried forward through the rest of the steps.

We sought to devise a strategy for the enantioselective synthesis of [3]-ladderanol by exploiting the symmetry of its tetracyclododecane skeleton and doing so with the direct installation of the linear 8-carbon alkyl chain in the desymmetrization step. Such an approach would allow for the introduction of chirality at a very late stage of the synthesis and provide the flexibility - not just in accessing both the enantiomers of [3]-ladderanol but also in modulating its structure for further studies.

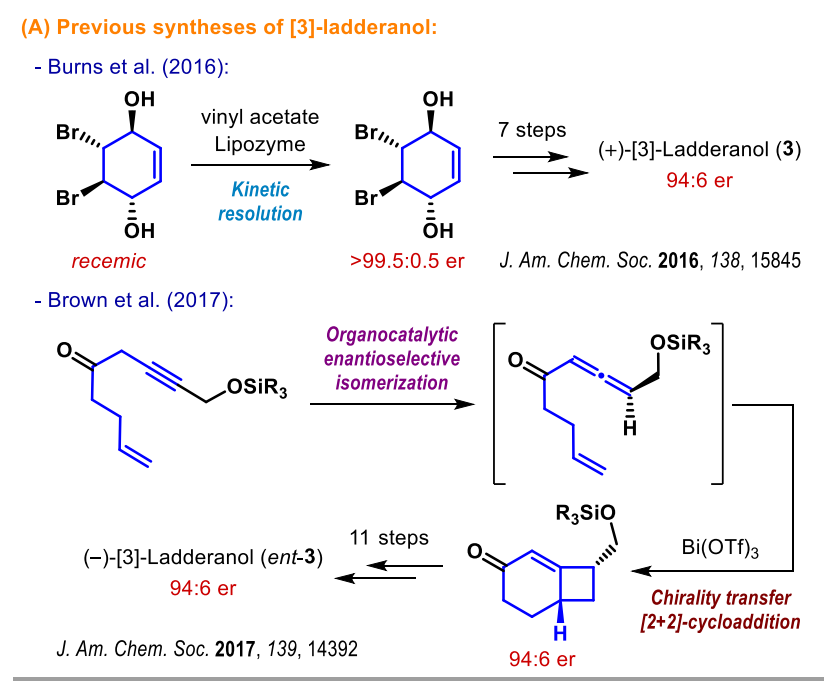

(B) Our strategy: A desymmetrization approach to [3]-ladderanol

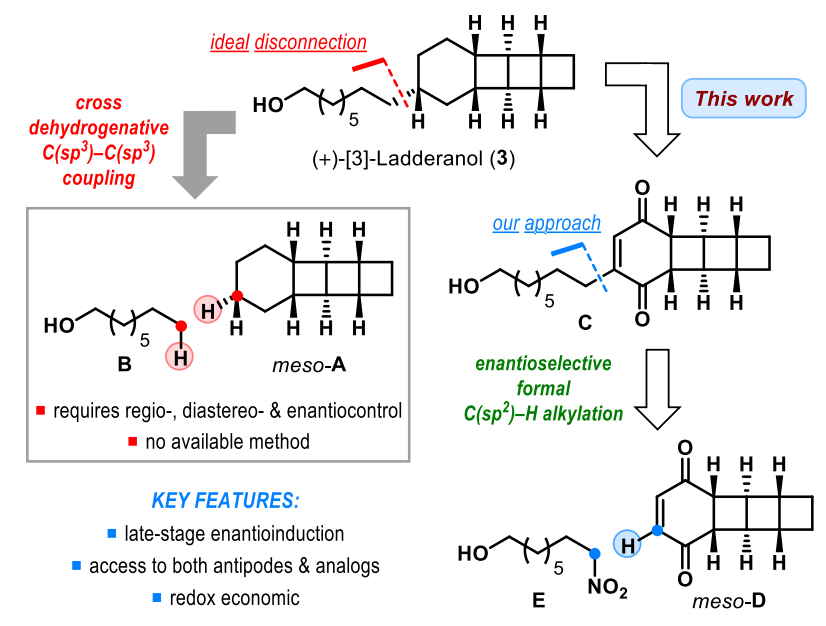

Scheme 1. Previous syntheses of [3]-ladderanol and our synthetic strategy

A direct desymmetrizing cross-dehydrogenative $\mathrm{C}\left(\mathrm{sp}^{3}\right)-\mathrm{C}\left(\mathrm{sp}^{3}\right)$ coupling ${ }^{[14]}$ between meso-tetracyclododecane $\mathbf{A}$ and 1-octanol B would constitute an ideal synthesis of [3]-ladderanol (Scheme 1B). However, a regio-, diastereo- and enantioselective coupling reaction of this kind poses a daunting challenge, and no such method is currently available at chemists' disposal.

Our deliberation along the same line of retrosynthetic analysis, instead, led us to a more realistic disconnection using tetracyclic meso-cyclohexenedione $\mathbf{D}$ as a synthon of $\mathbf{A}$ (Scheme 1B). We have previously developed enantioselective desymmetrizations of related prochiral or meso-enediones by organocatalytic formal $\mathrm{C}\left(\mathrm{sp}^{2}\right)-\mathrm{H}$ alkylation using nitroalkanes as the alkylating agent. ${ }^{[15]}$ Accordingly, we envisioned a direct coupling between 8-nitrooctan-1-ol $\mathbf{E}$ and meso-D for the enantioselective synthesis of the entire [3]-ladderanol framework $\mathbf{C}$. The remainder of the synthesis could then be accomplished by diastereoselective hydrogenation and deoxygenation of $\mathbf{C}$.

Herein we present a systematic implementation of this strategy, which eventually culminated in the total synthesis of both the enantiomers of [3]-ladderanol as well as an analog.

Although our previously developed desymmetrizing formal $\mathrm{C}\left(\mathrm{sp}^{2}\right)-\mathrm{H}$ alkylation of enediones ${ }^{[15]}$ formed the basis of this synthetic strategy, we were cognizant of the potential challenges posed by meso-D and related trans-cyclobutane fused polycyclic cyclohexenediones. In this reaction, stereocenters are generated remote from the reaction site ${ }^{[16]}$ and enantioposition-selective conjugate addition of nitronate is the key to its success (Scheme 2). Compared to both 2,2-disubstituted cyclopentene-1,3-diones (F) and meso-norbornenoquinones $(\mathbf{G})$, the substituents in trans-cyclobutane fused polycyclic cyclohexenediones (H) distance 
themselves further from the reactive centers and hence, from the catalyst. This structural feature renders the crucial enantioposition-selective addition even more challenging.

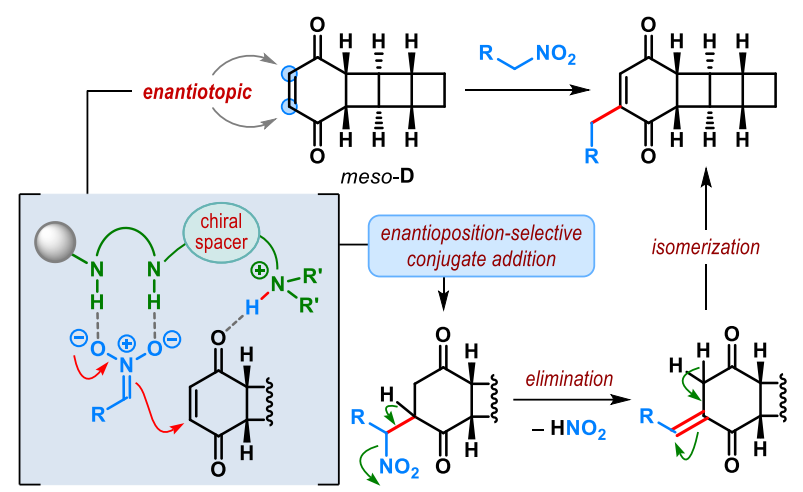

Requirement: Enantioposition-selective conjugate addition

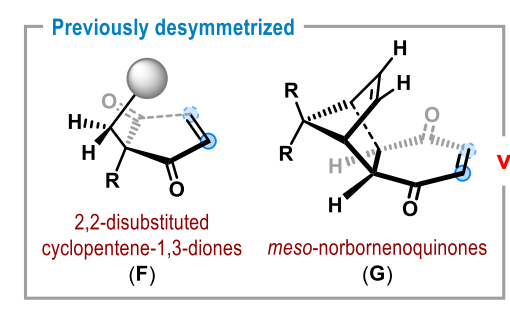

Advantage: Substituents on the ensuing stereocenter(s) are closer to the reaction sites

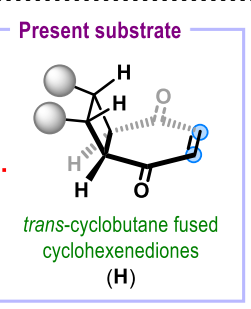

Challenge: Substituents are distant from the reaction sites

Scheme 2. Requirement for the key enantioselective formal $\mathrm{C}\left(\mathrm{sp}^{2}\right)-\mathrm{H}$ alkylation and the challenge posed by the present substrate

\section{Results and Discussion}

To identify the optimum catalyst and reaction conditions for the enantioselective formal $\mathrm{C}\left(\mathrm{sp}^{2}\right)-\mathrm{H}$ alkylation, we began our investigation with a model substrate - tricyclic meso-cyclohexenedione 5a, considering its easier accessibility and structural resemblance with $\mathbf{D}$ (Table 1). These cyclobutane fused meso-cyclohexenediones (5) can be prepared by [2+2]-photocycloaddition between cyclohexenedione 6 and an appropriate cis-alkene or alkyne 7, followed by dehydrogenation with $\mathrm{SeO}_{2}\left(\mathrm{Scheme} \mathrm{3).}{ }^{[17]} \mathrm{These}\right.$ [2+2]-photocycloaddition reactions of alkenes were seen to proceed usually in good yield and with complete exo-selectivity. Cyclohexenedione 6 can in turn be obtained from $p$-benzoquinone 9. ${ }^{[18]}$

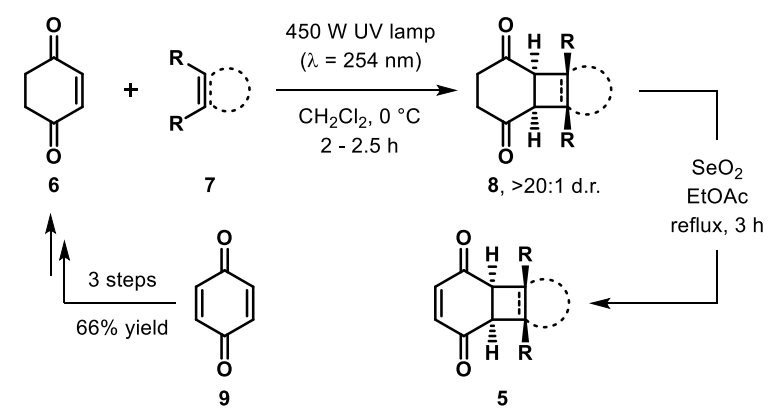

Scheme 3. Preparation of cyclobutane fused meso-cyclohexenediones 5

Initial optimization studies for $\mathrm{C}\left(\mathrm{sp}^{2}\right)-\mathrm{H}$ alkylation were carried out using nitromethane (10a) as the alkylating agent. ${ }^{[19]}$ When the reaction was carried out in $\mathrm{CHCl}_{3}$ with $10 \mathrm{~mol} \%$ quinine-derived bifunctional tertiary aminothiourea I as the catalyst ${ }^{[20]}$ and $\mathrm{Na}_{2} \mathrm{CO}_{3}$ as the terminal base, the desired C-H methylated product 11aa was formed in 41\% yield with a promising enantiomeric ratio (e.r.) of $92: 8$ (Table 1, entry 1). A slight improvement in yield and enantioselectivity was observed using the corresponding squaramide II as the catalyst $^{[21]}$ (entry 2). The enantioselectivity of this reaction turned out to be somewhat independent of the reaction medium (entries 4 6). However, lowering the initial concentration led to an enhancement in e.r. (entry 7). An attempt to improve the reaction rate and e.r. by increasing the catalyst loading proved futile (entry 8 ). Instead, a reduction in catalyst loading surprisingly resulted in the rate enhancement while maintaining the same level of enantioselectivity (entry 9). The reaction can be scaled up under these conditions, when 11aa was isolated in $75 \%$ yield with $95: 5$ e.r. (entry 10 ).

We were pleased to note that the catalyst and reaction conditions optimized for the formal $\mathrm{C}\left(\mathrm{sp}^{2}\right)-\mathrm{H}$ methylation of tricyclic meso-cyclohexenedione $\mathbf{5 a}$ could be successfully extrapolated to a variety of polycyclic meso-cyclohexenediones $\mathbf{5}$ (Table $2 \mathrm{~A}$ ). These examples not only include trans-cyclobutane fused tri- $\mathbf{( 5 a - d )}$ and tetracyclic substrates $(\mathbf{5 h}$-i) but also to bicyclic cyclohexenediones 
$(\mathbf{5 e - g})$. The products (11aa-ia) were generally isolated in moderate to good yield with modest enantioselectivity in most cases. Enantioenrichment of some of these products (11 ca, 11 ha) is possible with up to 98:2 e.r. by a single recrystallization. Most notably, our coveted tetracyclic meso-cyclohexenedione $\mathbf{5 i}$ could be desymmetrized efficiently, and the product $\mathbf{1 1}$ ia was obtained with good enantioselectivity, especially after recrystallization.

Overall, the level of enantioselectivity observed in this reaction is considerably lower compared to 2,2-disubstituted cyclopentene-1,3-diones $\mathbf{F}$ and norbornenoquinones $\mathbf{G}$ (Scheme 2). ${ }^{[15]}$ Barring 11ca which appears as an anomaly, the presence, or the absence of the trans-substituents on the fused cyclobutane ring imparted little influence on the enantioposition-selectivity (cf. 11ea and 11ia). This observation is in agreement with our anticipation and corroborated by the solid-state structure of the products $11 \mathrm{ha}$-ia (CCDC 2144865-6, Table 2), ${ }^{[22]}$ where the trans-substituents reside far from the reaction site. The product 11fa, bearing two cismethyl groups on the cyclobutane, on the other hand, was obtained with relatively higher e.r. Along the same line, the planar cyclobutene in $\mathbf{5 g}$ expectedly offered less steric crowding and resulted in $\mathbf{1 1}$ ga with much inferior e.r.

Table 1. Optimization of catalyst and reaction conditions for enantioselective formal $\mathrm{C}\left(\mathrm{sp}^{2}\right)-\mathrm{H}$ methylation of tricyclic meso-cyclohexenedione 5 a $^{\text {[a] }}$

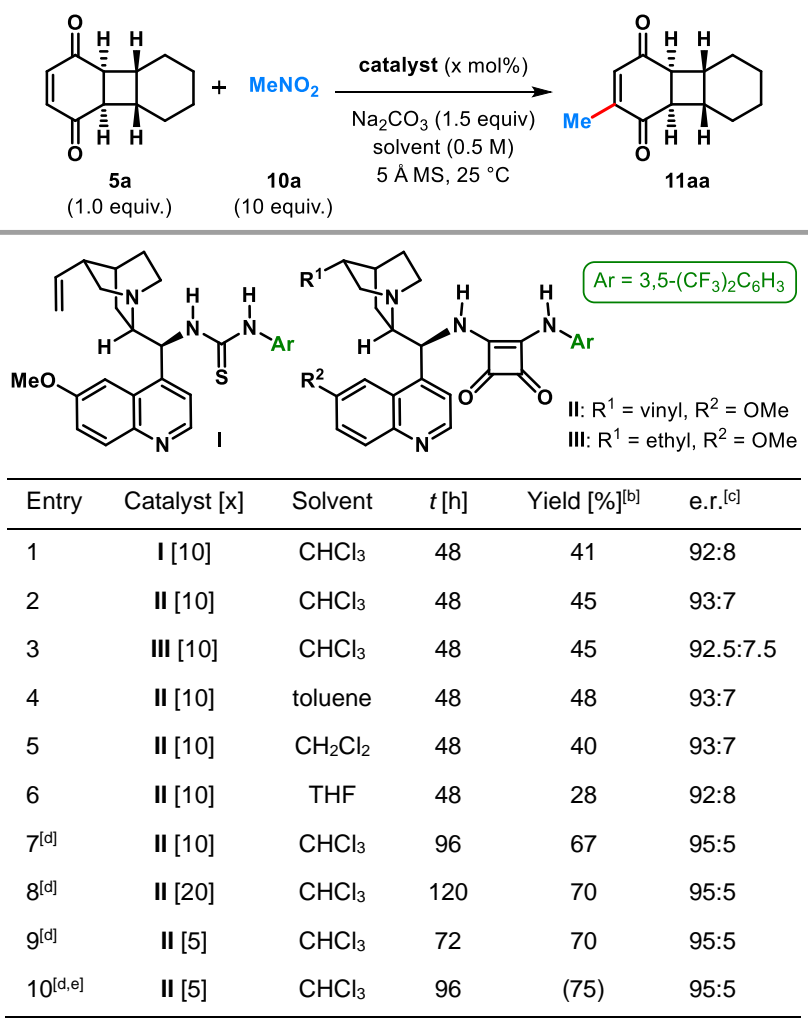

[a] Reactions were performed using 1.0 equiv of $\mathbf{5 a}$ and 10 equiv of $10 \mathrm{a}$ on a 0.1 mmol scale. [b] Yields were determined by ${ }^{1} \mathrm{H}$ NMR spectroscopy with $1,3,5$ trimethoxybenzene as the internal standard. Isolated yield is given in the parentheses. [c] Enantiomeric ratio (e.r.) as determined by HPLC analysis using a stationary phase chiral column. [d] Reaction at $0.1 \mathrm{M}$ initial concentration. [e] Reaction on a $0.3 \mathrm{mmol}$ scale.

The introduction of higher alkanes is also possible. However, as seen in our earlier studies, ${ }^{[15]}$ the reactions with higher nitroalkanes (10b-d) were found to be slower and proceeded with inferior enantioselectivities (Table 2B).

Nonetheless, the amenability of these reaction conditions to tetracyclic meso-cyclohexenedione $\mathbf{5 i}$ and the possibility of using higher nitroalkanes, especially $\mathbf{1 0 d}$, provided us the necessary incentive to attempt the direct installation of the linear 8-carbon alkyl chain through this enantioselective formal $\mathrm{C}\left(\mathrm{sp}^{2}\right)-\mathrm{H}$ alkylation reaction. Once again, our initial studies in this direction were focused on the model substrate $\mathbf{5 a}$. $^{[19]}$ Interestingly, the reaction of 5a with 0 -protected 8-nitrooctan-1-ols (10e-f) under our optimum reaction conditions (Table 2) resulted in the Michael adducts 12ae-af, ${ }^{[23]}$ which were isolated as a single diastereomer (Scheme $4 \mathrm{~A}$ ). The isolation of these Michael adducts momentarily gave rise to the possibility of accessing [3]-ladderanol through reductive denitration ${ }^{\text {[24] }}$ and deoxygenation by following a similar route with $5 \mathbf{i}$. Unfortunately, determination of absolute stereochemistry of 12ae through single crystal X-ray diffraction analysis (CCDC 2144867, Scheme 4A $)^{[22]}$ revealed the formation of the undesired diastereomer in this reaction and forced us to stick to our original plan (Scheme $1 \mathrm{~B}$ ). Although the desired $\mathrm{C}\left(\mathrm{sp}^{2}\right)-\mathrm{H}$ alkylated product $\mathbf{1 1}$ could be obtained with excellent enantioselectivity by treating 12 with a combination of KOAc and 18-crown-6, the abysmal yield of this twostep process prohibited us from applying it to the synthesis of [3]-ladderanol. 
Table 2. Scope of enantioselective formal $\mathrm{C}\left(\mathrm{sp}^{2}\right)-\mathrm{H}$ alkylation of cyclobutene-fused cyclohexenediones. ${ }^{[\mathrm{a}]}$

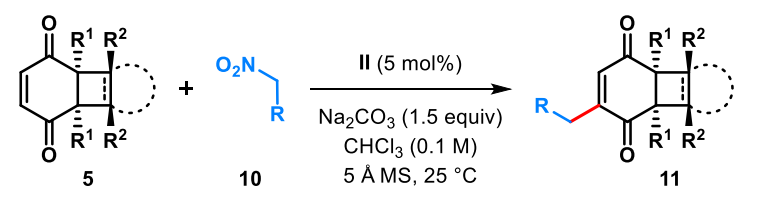

(A) Scope of Cyclohexenediones

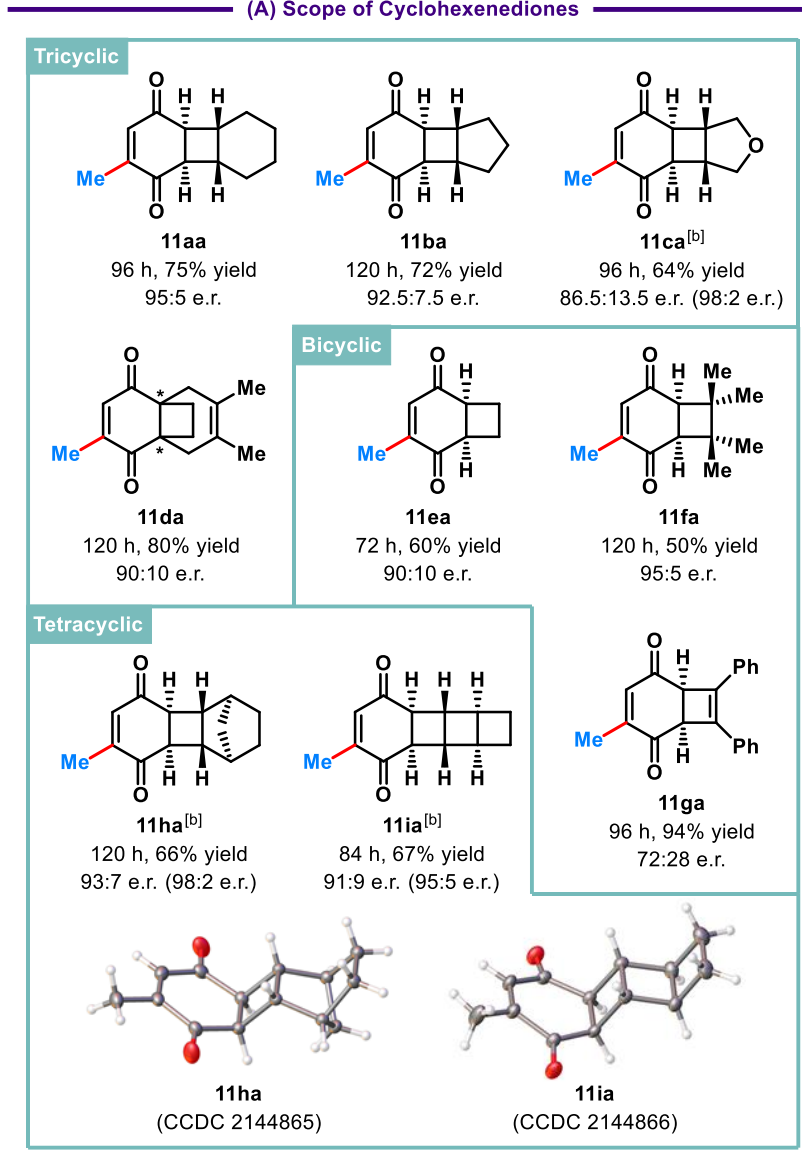

(B) Scope of Nitroalkanes

\begin{tabular}{llllcl} 
Entry & $\mathrm{R}(\mathbf{1 1})$ & $t[\mathrm{~h}]$ & Yield [\%] & e.r. \\
1 & $\mathrm{Me} \mathrm{(11ab)}$ & 120 & 23 & $74: 26$ \\
2 & $\mathrm{Ph}(\mathbf{1 1 a c})$ & 120 & 50 & $92: 8$ \\
3 & $\mathrm{CH}_{2} \mathrm{OTBS}(\mathbf{1 1} \mathbf{a d})$ & 120 & 62 & $90: 10$ \\
\hline
\end{tabular}

[a] Reactions were carried out on a $0.3 \mathrm{mmol}$ scale with either 10 equiv (10a-b) or 5 equiv (10c-d) of nitroalkanes and $50 \mathrm{mg} 5 \AA$ MS in a sealed vial. Yields correspond to the isolated yield after chromatographic purification. Enantiomeric ratio (e.r.) as determined by HPLC analysis using a stationary phase chiral column. [b] The numbers in the parentheses represent e.r. after a single recrystallization.

A detailed optimization of catalyst and reaction conditions resulted in a one-pot two-step protocol using two different bifunctional catalysts - one for each step. While the quinine-derived tertiary aminosquaramide II was efficient for the enantioposition-selective Michael addition, the elimination of $\mathrm{HNO}_{2}$ and isomerization were found to be slow under its influence. These latter steps proceeded relatively faster with the achiral tertiary aminourea IV (Scheme 4B). Under these conditions, 11ae was isolated in 75\% yield with $92: 8$ e.r. 

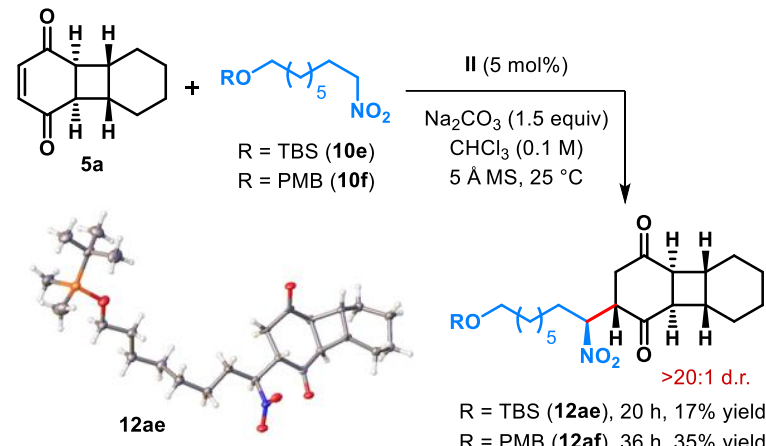

(CCDC 2144867)

$R=T B S(12 a e), 20 \mathrm{~h}, 17 \%$ yield

$\mathrm{R}=\mathrm{PMB}(\mathbf{1 2} \mathbf{a f}), 36 \mathrm{~h}, 35 \%$ yield

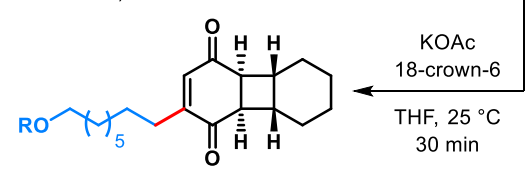

$R=$ TBS (11ae), 32\% yield, 99:1 e.r.

$\mathrm{R}=\mathrm{PMB}$ (11 af), 33\% yield, 98:2 e.r.

(B) Optimum conditions: $\quad$ II $(5 \mathrm{~mol} \%)$

$\mathrm{Na}_{2} \mathrm{CO}_{3}$ (1.5 equiv)

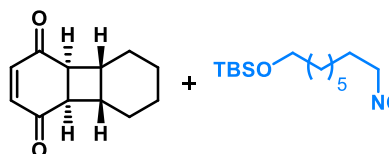

$\mathrm{CHCl}_{3}(0.1 \mathrm{M})$

$5 \mathrm{AMS}, 25^{\circ} \mathrm{C}, 24 \mathrm{~h}$

5a $(0.1 \mathrm{mmol})$

$10 \mathrm{e}(0.5 \mathrm{mmol})$

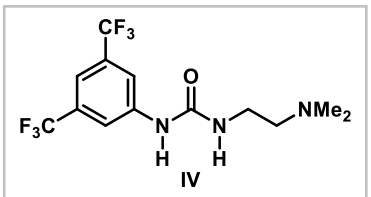

IV $(10 \mathrm{~mol} \%)$

$25^{\circ} \mathrm{C}, 96 \mathrm{~h}$

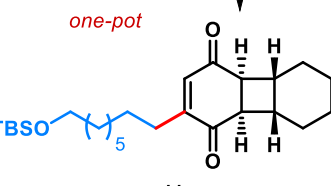

11 ae

$75 \%$ yield, $92: 8$ e.r.

Scheme 4. Installation of 8-carbon alkyl chain through enantioselective formal $\mathrm{C}\left(\mathrm{sp}^{2}\right)-\mathrm{H}$ alkylation

This one-pot two-step protocol was then applied to both the model substrate $5 \mathrm{a}$ (Table $3 \mathrm{~A}$ ) and the tetracyclic meso-cyclohexenedione $\mathbf{5 i}$ (Table 3B) with differently protected (10e-f) and even unprotected 8-nitrooctan-1-ol (10g). In the case of $O$ protected 8-nitrooctan-1-ols (10e-f), the products (11ae-af, 11ie-if) were generally obtained in good yield with decent enantioselectivities. However, lower yields and enantioselectivities were observed when the reactions were carried out with 8-nitrooctan-1-ol $10 \mathrm{~g}$. We were pleased to note the formation of the other product antipode with the same level of enantioselectivity in most cases when II was replaced by pseudoenantiomeric tertiary aminosquaramide $\mathbf{V}$, derived from quinidine. These reactions could be performed on a larger $(0.5 \mathrm{mmol})$ scale without significantly compromising the outcome. Although a large excess (5 equiv) of nitroalkanes $(\mathbf{1 0 e}-\mathbf{g})$ had to be used in these reactions for achieving a reasonable reaction rate, most of the unreacted nitroalkanes (3.6-3.9 equiv) could be recovered and reused. 
Table 3. Installation of 8-carbon alkyl chain: Scope ${ }^{[a]}$

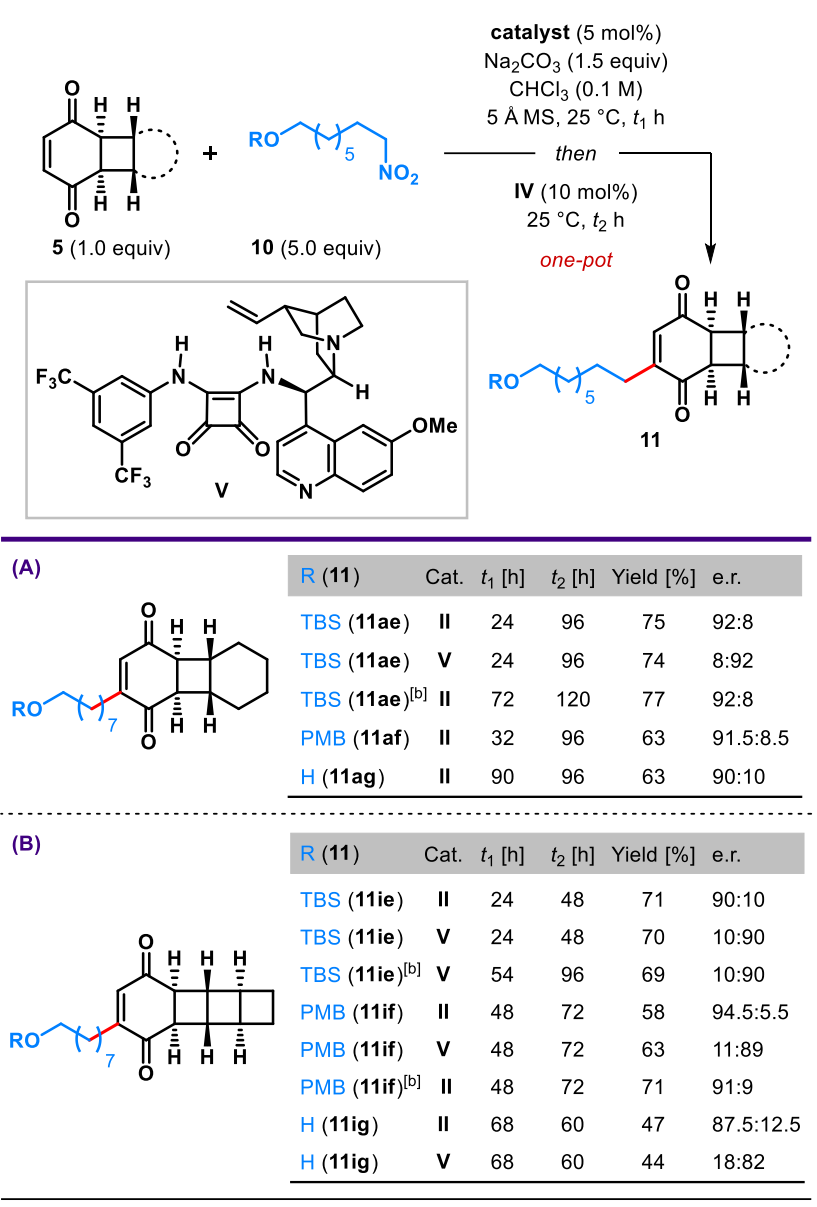

[a] Unless stated otherwise, reactions were carried out on either 0.2 (Table 3A) or 0.05 mmol scale (Table 3B). Yields correspond to the isolated yield after chromatographic purification. Enantiomeric ratio (e.r.) as determined by HPLC analysis using a stationary phase chiral column. [b] Reaction on a 0.5 mmol scale. TBS $=$ tert-Butyldimethylsilyl; $\mathrm{PMB}=p$-Methoxybenzyl .

After assembling the entire carbon-skeleton (11ie-ig) of [3]-ladderanol, only the complete reduction of the enedione functionality was left for achieving its total synthesis. We once again chose the model compound 11ae to probe our synthetic strategy. Our initial plan involved a diastereoselective hydrogenation of the olefin followed by ketone reduction and deoxygenation of the corresponding mesylate derivative. However, unexpected complications compelled us to abandon this and related strategies. ${ }^{[19]}$ We, instead, chose to adopt Burns' endgame ${ }^{[12]}$ for completing the total synthesis.

Accordingly, 11ae was transformed into 1,3-cyclohexadiene derivative $\mathbf{1 3}$ by Cu-TMEDA-mediated denitrogenation of the initially prepared bis-hydrazone (Scheme 5). Hydrogenation of the diene 13 under $\mathrm{Pd} / \mathrm{C}$ proceeded diastereoselectively and was accompanied by the deprotection of the TBS group to furnish [3]-ladderanol analog $\mathbf{1 4}$ as a single diastereomer in $72 \%$ isolated yield.

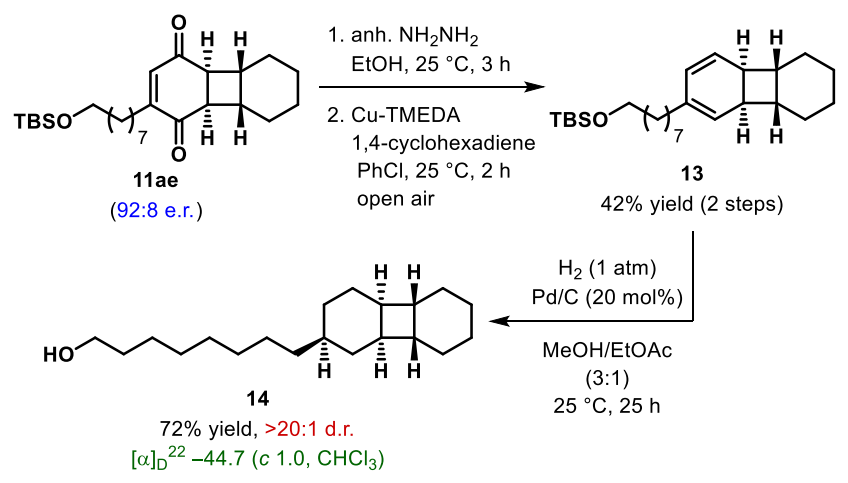

Scheme 5. Elaboration of desymmetrized tricyclic cyclohexenedione 11ae to [3]-ladderanol analog 14

With necessary groundworks done, the stage was set to complete the enantioselective synthesis of [3]-ladderanol. As discussed above (Scheme 3) and illustrated in Scheme 6, our key building block meso-cyclohexenedione $\mathbf{5 i}$ was assembled from cyclohexene1,4-dione $\mathbf{6}$ and bicyclohexene $7 \mathbf{i}$. Desymmetrization of $\mathbf{5 i}$ by enantioselective formal $\mathrm{C}\left(\mathrm{sp}^{2}\right)-\mathrm{H}$ alkylation with TBS-protected 8-nitrooctan-1-ol (10e) was accomplished using a sequential combination of tertiary aminosquaramide $\mathbf{V}$ and tertiary aminourea IV, as 
described in Table 3B. The product ent-11ie, having the entire carbon-skeleton of natural [3]-ladderanol with the correct stereochemistry at the fused ring junctions, thus obtained with 90:10 e.r., was then subjected to the two-step deoxygenation protocol developed by Burns et al. ${ }^{[12]}$ The resulting 1,3-cyclohexadiene derivative 15 was isolated in an overall yield of 54\% over two steps. Although the analogous 1,3-cyclohexadiene $\mathbf{1 3}$ could be hydrogenated diastereoselectively under Pd/C (see Scheme 5), subjecting 15 to this heterogeneous reaction conditions caused fragmentation of the strained tetracyclododecane ring to generate tricyclododecane derivative ent-14, albeit as a single diastereomer, in good yield. Hydrogenation under homogeneous conditions using Crabtree's catalyst $\left[\operatorname{Ir}(\mathrm{COD})\left(\mathrm{PCy}_{3}\right)(\mathrm{py})\right] \mathrm{PF}_{6}$, on the other hand, quantitatively converted 15 to the desired diastereomer $\mathbf{1 6}$ without affecting the ladderane motif. Deprotection of the TBS group with aqueous $\mathrm{HCl}$ concluded the total synthesis of $(+)$ [3]-ladderanol 3 - a component of the natural ladderane phospholipids.

Conversion of $\mathbf{3}$ to the corresponding p-bromophenyl carbamate $\mathbf{1 8}$ facilitated the growth of single crystals suitable for X-ray diffraction analysis (CCDC 2144892, Scheme 6) ${ }^{[22]}$ and further confirmed the absolute configuration of (+)-[3]-ladderanol.

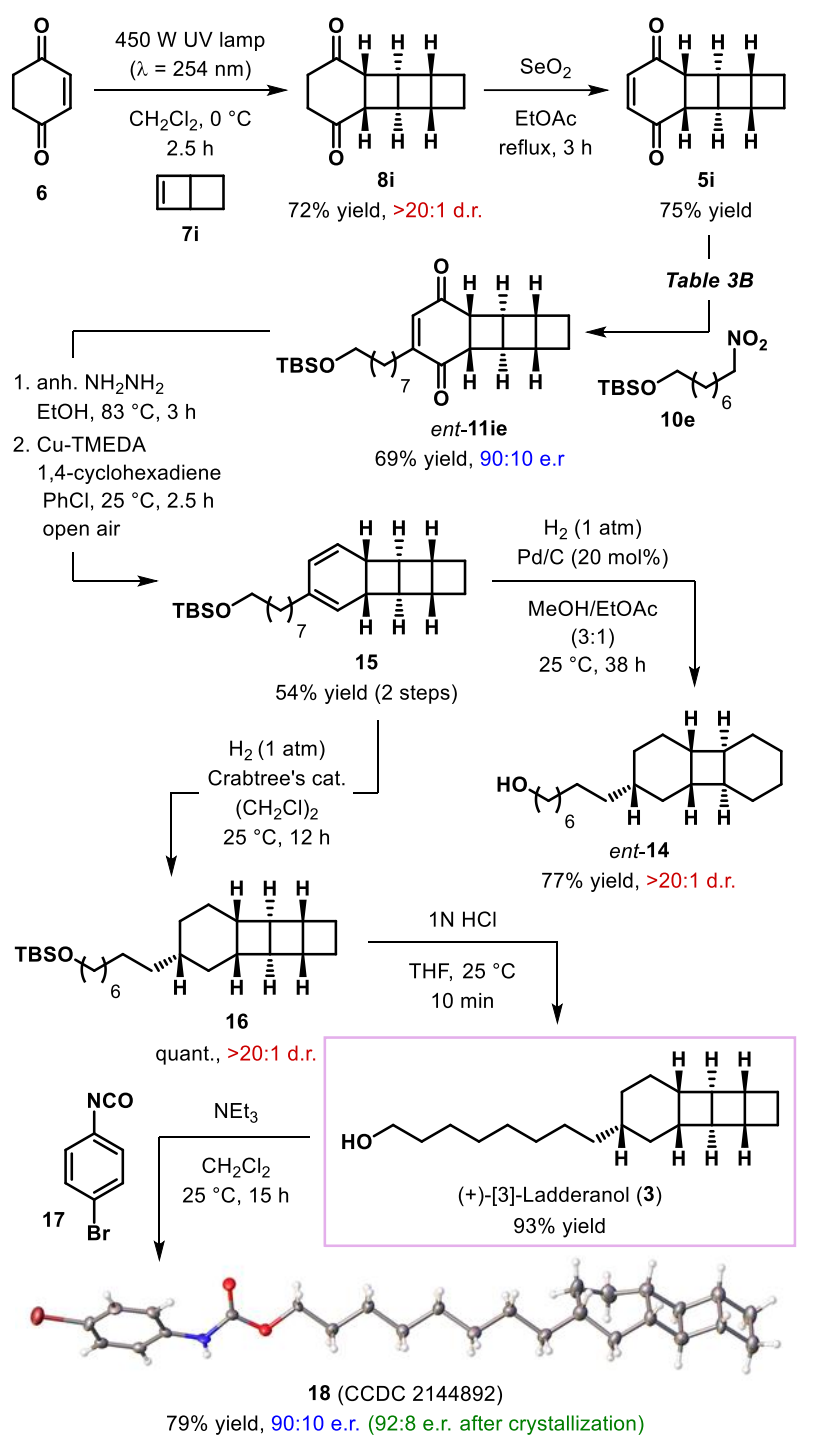

Scheme 6. Enantioselective total synthesis of (+)-[3]-ladderanol (3)

The introduction of chirality at a later stage renders flexibility to our synthetic route. We chose to demonstrate the advantage of this modular approach by synthesizing the non-natural antipode of [3]-ladderanol ent-3 (Scheme 7). This time we decided to use the PMB-protected desymmetrized tetracyclic cyclohexenedione 11if, an intermediate in Burns' synthesis, ${ }^{[12]}$ which we could prepare with 91:9 e.r. (see Table 3B). Starting from 11if, following Burns' protocol, ${ }^{[12]}(-)$-[3]-ladderanol ent-3 was synthesized in $43 \%$ yield over four steps. As shown with (+)-[3]-ladderanol (Scheme 6), in this case also enantioenrichment of the corresponding p-bromophenyl carbamate (ent-18) could be achieved after a single recrystallization. 


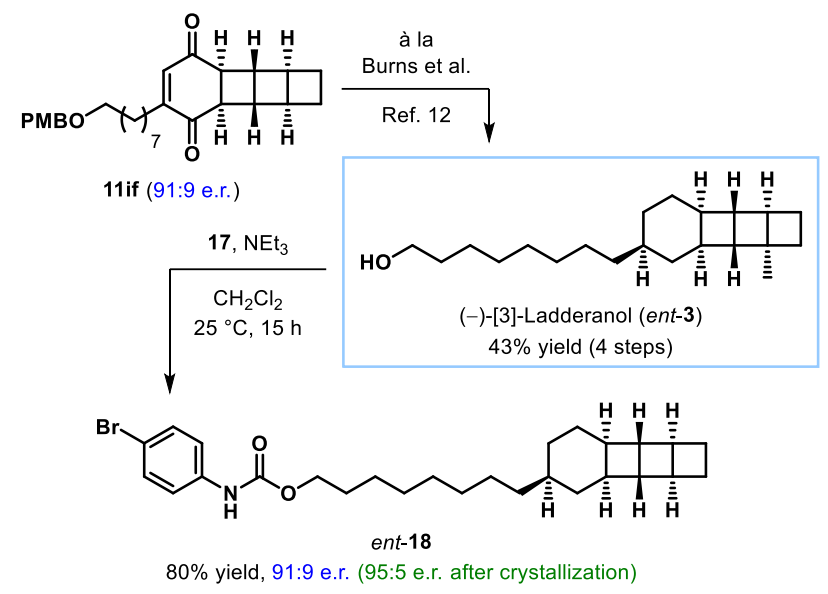

Scheme 7. Elaboration of 11if to (-)-[3]-ladderanol (ent-3)

\section{Conclusion}

In conclusion, we have accomplished an enantioselective total synthesis of [3]-ladderanol. Our synthetic strategy rests on a conceptually novel direct enantioselective assembly of the linear alkyl chain with the tetracyclododecane core of [3]-ladderanol. In practice, this intricate task is realized through a desymmetrizing formal $\mathrm{C}\left(\mathrm{sp}^{2}\right)-\mathrm{H}$ alkylation of tetracyclic meso-cyclohexenedione - the tetracyclododecane core at a higher oxidation state. This one-pot two-step $\mathrm{C}-\mathrm{C}$ bond forming transformation, utilizing $\mathrm{O}$-protected 8-nitrooctan-1-ol as an air and moisture-stable alkyl sources, is catalyzed by a sequential combination of a chiral tertiary aminosquaramide and an achiral tertiary aminourea. This is the first time an enantioselective desymmetrization strategy is applied to the synthesis of [3]-ladderanol. The late-stage introduction of chirality offers flexibility to this already concise route, which allowed for the synthesis of both the enantiomers of [3]-ladderanol as well as its analog with a good level of enantioselectivity.

\section{Acknowledgements}

This work is funded by Science and Engineering Research Board (SERB), New Delhi [Grant No. EMR/2016/005045]. S.R. acknowledges the Ministry of Education, Government of India for his doctoral fellowship through the Prime Minister's Research Fellowship (PMRF) scheme. S.M. thanks Council of Scientific and Industrial Research (CSIR) for his doctoral fellowship. We sincerely thank Prof. N. D. Pradeep Singh (Department of Chemistry, Indian Institute of Technology Kharagpur) for helpful discussion and for allowing us to use the photoreaction set-up in his laboratory for our preliminary studies. High resolution mass spectra (HR-MS) were recorded on an equipment procured under the Department of Science and Technology (DST)-FIST grant [Grant No. SR/FST/CS II-040/2015]. We thank Mr. Sujit Kamilya, Mr. Sounak Ghosh, and Dr. Sayed Muktar Hossain (Solid State and Structural Chemistry Unit, IISc, Bangalore) for their help with the X-ray diffraction analyses.

\section{References}

[1] a) J. T. Mohr, M. R. Krout, B. M. Stoltz, Nature 2008, 455, 323-332; b) M. S. Taylor, E. N. Jacobsen, Proc. Natl. Acad. Sci. U. S. A. 2004, 101, 5368-5373.

[2] J. S. Sinninghe Damsté, M. Strous, W. I. C. Rijpstra, E. C. Hopmans, J. A. J. Geenevasen, A. C. T. van Duin, L. A. van Niftrik, M. S. M. Jetten, Nature 2002, 419, 708-712.

[3] a) I. Novak, J. Phys. Chem. A 2008, 112, 10059-10063; b) M. A. Miller, J. M. Schulman, THEOCHEM 1988, 163, $133-141$.

[4] a) A. Dietl, C. Ferousi, W. J. Maalcke, A. Menzel, S. de Vries, J. T. Keltjens, M. S. M. Jetten, B. Kartal, T. R. M. Barends, Nature 2015, 527, 394-397; b) B. Kartal, N. M. de Almeida, W. J. Maalcke, H. J. M. Op den Camp, M. S. M. Jetten, J. T. Keltjens, FEMS Microbiol. Rev. 2013, 37, 428-461; c) B. Kartal, W. J. Maalcke, N. M. de Almeida, I. Cirpus, J. Gloerich, W. Geerts, H. J. M. Op den Camp, H. R. Harhangi, E. M. Janssen-Megens, K.-J. Francoijs, H. G. Stunnenberg, J. T. Keltjens, M. S. M. Jetten, M. Strous, Nature 2011, 479, 127130; d) M. S. M. Jetten, L. van Niftrik, M. Strous, B. Kartal, J. T. Keltjens, H. J. M. Op den Camp, Crit. Rev. Biochem. Mol. Biol. 2009, 44, 65-84; e) J. G. Kuenen, Nat. Rev. Microbiol. 2008, 6, 320-326; f) L. A. van Niftrik, J. A. Fuerst, J. S. S. Damsté, J. G. Kuenen, M. S. M. Jetten, M. Strous, FEMS Microbiol. Lett. 2004, 233, 7-13.

[5] a) F. R. Moss, S. R. Shuken, J. A. M. Mercer, C. M. Cohen, T. M. Weiss, S. G. Boxer, N. Z. Burns, Proc. Natl. Acad. Sci. USA 2018, 115, 9098-9103; b) W. R. L. van der Star, C. Dijkema, P. de Waard, C. Picioreanu, M. Strous, M. C. M. van Loosdrecht, Appl. Microbiol. Biotechnol. 2010, 86, 311-317.

[6] a) W. R. L. van der Star, A. I. Miclea, U. G. J. M. van Dongen, G. Muyzer, C. Picioreanu, M. C. M. van Loosdrecht, Biotechnol. Bioeng. 2008, 101, 286-294; b) M. Strous, J. J. Heijnen, J. G. Kuenen, M. S. M. Jetten, Appl. Microbiol. Biotechnol. 1998, 50, 589-596.

[7] a) V. Raghavan, J. L. Johnson, D. F. Stec, B. Song, G. Zajac, M. Baranska, C. M. Harris, N. D. Schley, P. L. Polavarapu, T. M. Harris, J. Nat. Prod. 2018, 81, 2654-2666; b) H. A. Boumann, E. C. Hopmans, I. Van De Leemput, H. J. M. Op den Camp, J. Van De Vossenberg, M. Strous, M. S. M. Jetten, J. S. Sinninghe Damsté, S. Schouten, FEMS Microbiol. Lett. 2006, 258, 297-304; c) J. S. Sinninghe Damsté, 
W. I. C. Rijpstra, J. A. J. Geenevasen, M. Strous, M. S. M. Jetten, FEBS J. 2005, 272, 4270-4283; d) J. S. Sinninghe Damsté, W. I. C. Rijpstra, M. Strous, M. S. M. Jetten, O. R. P. David, J. A. J. Geenevasen, J. H. van Maarseveen, Chem. Commun. 2004, $2590-2591$.

[8] For selected examples, see: a) H. Hopf, H. Greiving, P. G. Jones, P. Bubenitschek, Angew. Chem., Int. Ed. Engl. 1995, 34, 685-687; b) G. Mehta, M. B. Viswanath, G. N. Sastry, E. D. Jemmis, D. S. K. Reddy, A. C. Kunwar, Angew. Chem., Int. Ed. Engl. 1992, 31, 14881490; c) H.-D. Martin, B. Mayer, M. Pütter, H. Höchstetter, Angew. Chem., Int. Ed. Engl. 1981, 20, 677-678; d) H.-D. Martin, M. Hekman, Angew. Chem., Int. Ed. Engl. 1973, 12, 572-574; e) M. Avram, I. G. Dinulescu, E. Marica, G. Mateescu, E. Sliam, C. D. Nenitzescu, Chem. Ber. 1964, 97, 382-389. For reviews, see: f) H. D. Nouri, J. D. Tantillo, Curr. Org. Chem. 2006, 10, 2055-2074; g) H. Hopf, Angew. Chem., Int. Ed. 2003, 42, 2822-2825; h) W. Adam, O. De Lucchi, Angew. Chem., Int. Ed. 1980, 19, 762-779.

[9] For a review, see: E. N. Hancock, M. K. Brown, Chem.-Eur. J. 2021, 27, 565-576.

[10] V. Mascitti, E. J. Corey, J. Am. Chem. Soc. 2004, 126, 15664-15665.

[11] V. Mascitti, E. J. Corey, J. Am. Chem. Soc. 2006, 128, 3118-3119.

[12] J. A. M. Mercer, C. M. Cohen, S. R. Shuken, A. M. Wagner, M. W. Smith, F. R. Moss III, M. D. Smith, R. Vahala, A. Gonzalez-Martinez, S. G. Boxer, N. Z. Burns, J. Am. Chem. Soc. 2016, 138, 15845-15848.

[13] a) E. N. Hancock, E. L. Kuker, D. J. Tantillo, M. K. Brown, Angew. Chem., Int. Ed. 2020, 59, 436-441; b) N. J. Line, B. P. Witherspoon, E. N. Hancock, M. K. Brown, J. Am. Chem. Soc. 2017, 139, 14392-14395.

[14] For selected reviews, see: a) B. V. Varun, J. Dhineshkumar, K. R. Bettadapur, Y. Siddaraju, K. Alagiri, K. R. Prabhu, Tetrahedron Lett. 2017, 58, 803-824; b) C. S. Yeung, V. M. Dong, Chem. Rev. 2011, 111, 1215-1292; c) C.-J. Li, Acc. Chem. Res. 2009, 42, 335-344.

[15] a) R. Sarkar, S. Mukherjee, Org. Lett. 2016, 18, 6160-6163; b) M. S. Manna, S. Mukherjee, J. Am. Chem. Soc. 2015, 137, $130-133$.

[16] For selected examples of such remote stereocontrol, see: a) Y. Lou, J. Wei, M. Li, Y. Zhu, J. Am. Chem. Soc. 2022, 144, 123-129; b) G. R. Genov, J. L. Douthwaite, A. S. K. Lahdenperä, D. C. Gibson, R. J. Phipps, Science 2020, 367, 1246-1251; c) H. Wu, Q. Wang, J. Zhu, Angew. Chem., Int. Ed. 2018, 57, 2721-2725; d) H. Shi, A. N. Herron, Y. Shao, Q. Shao, J.-Q. Yu, Nature 2018, 558, 581-585; e) B. Su, T.-G. Zhou, P.-L. Xu, Z.-J. Shi, J. F. Hartwig, Angew. Chem., Int. Ed. 2017, 56, 7205-7208; f) W. Luo, L. Lin, Y. Zhang, X. Liu, X. Feng, Org. Lett. 2017, 19, 3374-3377; g) Z. Huang, X. Huang, B. Li, C. Mou, S. Yang, B.-A. Song, Y. R. Chi, J. Am. Chem. Soc. 2016, 138, 7524-7527; h) M. S. Manna, R. Sarkar, S. Mukherjee, Chem.-Eur. J. 2016, 22, 14912-14919; i) F. Zhou, C. Tan, J. Tang, Y.-Y. Zhang, W.-M. Gao, H.-H. Wu, Y.-H. Yu, J. Zhou, J. Am. Chem. Soc. 2013, 135, 10994-10997; j) C. A. Lewis, J. L. Gustafson, A. Chiu, J. Balsells, D. Pollard, J. Murry, R. A. Reamer, K. B. Hansen, S. J. Miller, J. Am. Chem. Soc. 2008, 130, 16358-16365; k) D. R. Cefalo, A. F. Kiely, M. Wuchrer, J. Y. Jamieson, R. R. Schrock, A. H. Hoveyda, J. Am. Chem. Soc. 2001, 123, 3139-3140.

[17] M. Oda, H. Oikawa, Y. Kanao, A. Yamamuro, Tetrahedron Lett. 1978, 19, 4905-4908.

[18] M. Oda, T. Kawase, T. Okada, T. Enomoto, Org. Synth. 1996, 73, 253.

[19] For details, see the Supporting Information.

[20] For pioneering work on bifunctional thiourea catalysis, see: a) T. Okino, Y. Hoashi, Y. Takemoto, J. Am. Chem. Soc. 2003, 125, 1267212673. For seminal works on cinchona alkaloid-based bifunctional thiourea catalysts, see: b) S. H. McCooey, S. J. Connon, Angew. Chem., Int. Ed. 2005, 44, 6367-6370; c) J. Ye, D. J. Dixon, P. S. Hynes, Chem. Commun. 2005, 4481-4483; d) B. Vakulya, S. Varga, A. Csámpai, T. Soós, Org. Lett. 2005, 7, 1967-1969; e) B.-J. Li, L. Jiang, M. Liu, Y.-C. Chen, L.-S. Ding, Y. Wu, Synlett 2005, 603-606.

[21] For seminal work on bifunctional squaramide catalysts, see: J. P. Malerich, K. Hagihara, V. H. Rawal, J. Am. Chem. Soc. 2008, 130, 14416-14417.

[22] Deposition Numbers 2144865-2144867, 2144892 (for 11ha, 11ia, 12ae and 18, respectively) contains the supplementary crystallographic data for this paper. These data are provided free of charge by the joint Cambridge Crystallographic Data Centre and Fachinformationszentrum Karlsruhe Access Structures service.

[23] For selected examples of catalytic enantioselective nitro-Michael reactions, see: a) D. Trubitsõn, J. Martõnova, M. Kudrjašova, K. Erkman, I. Järving, T. Kanger, Org. Lett. 2021, 23, 1820-1824; b) S. Bhunia, S. Chaudhuri, A. Bisai, Chem.-Eur. J. 2017, 23, 1123411238; c) M. Kawada, K. Nakashima, S.-i. Hirashima, A. Yoshida, Y. Koseki, T. Miura, J. Org. Chem. 2017, 82, 6986-6991; d) K. L. Jensen, C. F. Weise, G. Dickmeiss, F. Morana, R. L. Davis, K. A. Jørgensen, Chem.-Eur. J. 2012, 18, 11913-11918; e) W. Li, H. Liu, X. Jiang, J. Wang, ACS Catal. 2012, 2, 1535-1538; f) S. Rajkumar, K. Shankland, G. D. Brown, A. J. A. Cobb, Chem. Sci. 2012, 3, 584588; g) W. Yang, D.-M. Du, Org. Lett. 2010, 12, 5450-5453; h) C. Rabalakos, W. D. Wulff, J. Am. Chem. Soc. 2008, 130, 13524-13525; i) E. Reyes, H. Jiang, A. Milelli, P. Elsner, R. G. Hazell, K. A. Jørgensen, Angew. Chem., Int. Ed. 2007, 46, 9202-9205; j) S. B. Tsogoeva, S. B. Jagtap, Synlett 2004, 2624-2626. For a review, see: k) L. S. Aitken, N. R. Arezki, A. Dell'Isola, A. J. A. Cobb, Synthesis 2013, 45, 2627-2648.

[24] a) A. Kamimura, N. Ono, Bull. Chem. Soc. Jpn. 1988, 61, 3629-3635; b) N. Ono, H. Miyake, A. Kamimura, I. Hamamoto, R. Tamura, A. Kaji, Tetrahedron 1985, 41, 4013-4023. 